Gynäkologe 2015 · 48:632

DOI 10.1007/s00129-015-3766-0

Online publiziert: 20. August 2015

(c) Springer-Verlag Berlin Heidelberg 2015

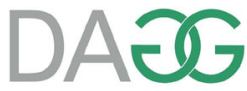

Deutsche Akademie

für Gynäkologie und Geburtshilfe

Olaf Ortmann für das Kuratorium und den Beirat der DAGG

\title{
Die Deutsche Akademie für Gynäkologie und Geburtshilfe (DAGG)
}

der DAGG wird alle 2 Jahre, alternierend zum wissenschaftlichen Kongress der DGGG, ein Weiterbildungskongress der DAGG stattfinden (www.dggg.de, 17.-19.09.2015, 7. Kongress des Forum Operative Gynäkologie (FOG) und 4. Kongress der DAGG, Berlin).

\section{I) Eine kontinuierliche} Weiterbildung ist sinnvoll und hilfreich

Neben Fach und Schwerpunktweiterbildung und der täglichen klinischen Arbeit ist eine kontinuierliche Weiterbildung sinnvoll und hilfreich. In der Rubrik „CME-Zertifizierte Fortbildung“ werden qualitativ hochwertige Artikel zu praktisch relevanten Themen publiziert. Nach der Lektüre der Beiträge kann der Leser sein erworbenes Wissen überprüfen und online CMEPunkte erhalten. Die Rubrikherausgeber haben für die kommenden Jahre Themen zusammengestellt, die sich an der Weiterbildungsordnung des Fachs orientieren. Die regelmäßige Lektüre der Artikel stellt somit ein ideales weiterbildungsbegleitendes Instrument dar. Dieses entspricht der Zielsetzung der
DAGG, die die Rubrik nachdrücklich unterstützt. Daher finden Sie ab dieser Ausgabe den Zusatz „Empfohlen von der DAGG“ bei den CME-Artikeln.

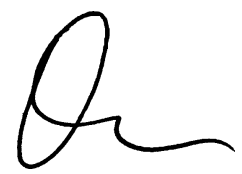

Prof. Dr. Olaf Ortmann

\section{Korrespondenzadresse}

\section{Prof. Dr. O. Ortmann}

Klinik für Frauenheilkunde und Geburtshilfe, Universität Regensburg

Landshuter Straße 65, 93053 Regensburg,

Deutschland

olaf.ortmann@klinik.uni-regensburg.de

Interessenkonflikt. O. Ortmann gibt an, dass kein Interessenkonflikt besteht. 